\title{
Le Rwanda, une obsession française
}

\author{
Boubacar Boris Diop
}

\begin{abstract}
Citer cet article : Diop Boubacar Boris (2021), "Le Rwanda, une obsession française », Revue d'Histoire Contemporaine de l'Afrique, Dossier : au-delà du rapport Duclert, 63-72, en ligne. URL : https://oap.unige.ch/journals/rhca/article/view/rwandadiop
\end{abstract}

Mise en ligne : 08 novembre 2021

DOI : https://doi.org/10.51185/journals/rhca.2021.e581

Le rapport de la Commission de recherche sur les archives françaises relatives au Rwanda et au génocide des Tutsi remis le 26 mars 2021 au président Emmanuel Macron n'est pas le premier à se pencher sur les responsabilités de la France. En décembre 1998, la mission Quilès publiait, après huit mois d'enquête, un volumineux document sur une éventuelle implication de Paris dans le génocide des Tutsi au Rwanda. Le choix des députés de s'en tenir à un simple travail d'information laissait présager d'une conclusion écrite à l'avance: ils invitaient les Rwandais à accepter d'être responsables de leur propre histoire, tout en égratignant au passage les Nations unies et, par petites allusions perfides, I'Amérique de Bill Clinton. De façon tout aussi prévisible, le rôle de Paris dans la tragédie était complètement nié. Tout juste reprochait-on au régime de François Mitterrand des erreurs d'appréciation dues à une trop grande proximité avec le régime corrompu, raciste et violent de Juvénal Habyarimana.

Six ans plus tard, la commission d'enquête citoyenne initiée par des associations (Survie et Aircrige entre autres) et des personnalités de la société civile, pointait du doigt les lourds silences et les contre-vérités de parlementaires français manifestement plombés par la raison d'État.

$\mathrm{Si}$ on ajoute à ces investigations celle du groupe d'experts présidé par l'historien Vincent Duclert, cela fait tout de même beaucoup de rapports, en trois décennies, sur un pays africain que la France n'a jamais formellement colonisé et où elle n'a d'ailleurs pris pied qu'au milieu des années 1970.

En fait, si l'opinion française n'a jamais vraiment pris au sérieux le génocide des Tutsi au Rwanda, l'establishment politico-médiatique, lui, a tendance depuis le début à l'analyser sous l'angle d'une possible complicité de l'État français avec ses planificateurs. Et l'on peut dire qu'ici deux visions de la tragédie s'affrontent habituellement.

Pour certains intellectuels et hommes politiques, le mot "génocide » est si chargé qu'ils sont révulsés par l'idée que la France ait pu être mêlée, si peu que ce soit, à la 
destruction des Tutsi du Rwanda. Cette hantise les a souvent conduits au cours des dernières années à des excès de langage dépassant parfois l'entendement. On rappelle souvent le fameux «Dans ces pays-là, un génocide ça n'est pas trop important » de Mitterrand rapporté par le journaliste Patrick de Saint-Exupéry ${ }^{1}$. Au-delà de l'incroyable obscénité du propos, il est facile de deviner les états d'âme d'un vieil homme effaré, comme désireux de se convaincre à tout prix qu'il n'a pas pu se compromettre - entraînant son pays avec lui - dans un "vrai génocide ", comparable à celui commis contre les Juifs par Adolf Hitler. Cette déclaration est surtout un des symptômes d'une discrète obsession française pour le Rwanda qui a donné lieu à bien des crises de nerfs. Insulter les victimes du génocide de 1994 est en effet devenu peu à peu une sorte de rituel d'exorcisme chez bien des leaders d'opinion de l'Hexagone. Aucun crachat n'est jugé de trop dès lors que l'animalisation des victimes peut aider la "patrie des droits de l'homme » à se sentir moins coupable. L'historien Nicolas Bancel a répertorié quelques déclarations de la même veine que celle de Mitterrand. Charles Pasqua, par exemple, invité du journal télévisé de France 2 en juin 1994, ne se gêne pas pour faire remarquer au journaliste l'invitant à réagir aux tueries en cours au pays des mille collines: "Vous savez, il faut bien comprendre que pour ces gens-là, le caractère horrible de ce qui s'est passé n'a pas la même valeur que pour nous². » Un mois plus tard, l'académicien Jean d'Ormesson «embarqué » par l'opération Turquoise du côté de Gikongoro, en revient frétillant d'aise et s'épanche en ces termes dans Le Figaro des 19 et 20 juillet 1994 : "Sortez vos mouchoirs: il va y avoir des larmes. Âmes sensibles, s'abstenir : le sang va couler à flot sous les coups de machette ${ }^{3}$. " "Partout... le sang a coulé à flots et coulera sans doute encore. Ce sont des massacres grandioses dans des paysages sublimes ${ }^{4}$. » Oui, on a bien lu, même si trente ans après, il reste difficile de croire que ces mots-là ont réellement pu être écrits dans un respectable quotidien parisien à grand tirage...

Sans vouloir retourner le couteau dans la plaie, on est bien obligé de se souvenir aussi des innombrables plaisanteries de mauvais goût inspirées à des caricaturistes ou chroniqueurs de l'audiovisuel par les souffrances du peuple rwandais. Ainsi, en 2014, beaucoup de radios - et peut-être quelques télévisions - françaises se sont regroupées au dernier étage d'un grand hôtel de Kigali pour rendre compte du vingtième anniversaire du génocide. Invité par elles à plusieurs reprises, j'ai été très surpris de voir à quel point ces professionnels des médias faisaient fi du contexte de recueillement, étant surtout occupés à tirer à boulets rouges sur le « dictateur Kagame ». II est vrai que la tension était brusquement montée entre Kigali et Paris à la veille de cette commémoration. François Hollande avait d'ailleurs, à la stupéfaction de nombreux invités déjà sur place, refusé de s'y faire représenter et Kagame lui avait répondu en glissant dans un discours en anglais son malicieux « les faits sont têtus », qui mit facilement les ricaneurs de son côté.

Comment expliquer que le Rwanda soit le seul des pays dits « du champ » avec lequel la France ait une relation aussi heurtée et passionnelle ? C'est sans doute dû au fait qu'elle n'est pas habituée à se voir reprocher les crimes de la conquête coloniale ou les sanglantes

\footnotetext{
1 Patrick de Saint-Exupéry, "France-Rwanda : un génocide sans importance », Le Figaro, 12 janvier 1998.

2 Bancel Nicolas (2002), "Les médias français face au Rwanda. De l'intervention française de 1990 au génocide », Africultures, 30, en ligne. URL : http://africultures.com/les-medias-francais-face-au-rwanda-1471/ (consulté le 11 octobre 2021).

3 Jean d'Ormesson, "J'ai vu le malheur en marche », Le Figaro, 19 juillet 1994.

4 Jean d'Ormesson, «La drôle d'odeur de l'église de Kibuye », Le Figaro, 20 juillet 1994.
} 
dérives de la Françafrique. Elle a toujours su garder une si grande influence dans son exempire colonial qu'elle est aujourd'hui encore persuadée d'y avoir agi pour le seul bénéfice des populations africaines. Une telle disposition d'esprit rappelle, soit dit au passage, la "doctrine des bonnes intentions " typique, selon Noam Chomsky, de l'impérialisme américain et, plus largement, occidental ${ }^{5}$. II faut bien se rendre compte que malgré ses nombreux massacres coloniaux - Madagascar, Sétif, Cameroun - et les centaines de milliers de morts de la guerre d'Algérie, la France continue à se sentir suffisamment innocente pour faire voter en février 2005 une « loi sur les aspects positifs de la colonisation ».

Voilà pourquoi elle a du mal à comprendre qu'on l'incrimine dans la campagne d'extermination des Tutsi en 1994 alors qu'elle n'a pas brandi une seule machette ou tiré le moindre coup de feu. Mais pour qui connaît I'histoire contemporaine, il est normal que le Rwanda fasse battre si fort son cœur : c'est qu'ici on parle d'un génocide, le dernier du $x^{\mathrm{e}}$ siècle, universellement reconnu comme tel. Le seul fait d'en être tenu pour complice suffit à plonger quiconque dans un abîme d'infamie.

En vérité, tout aurait été plus simple si la France avait pu, comme elle sait si bien le faire, écrire cette histoire africaine-là à sa guise.

La victoire politique et militaire du Front patriotique rwandais (FPR) I'a privée cette foisci de toute possibilité de manipulation des faits. Il est certain qu'avec un régime rwandais aux ordres de Paris - ou dépendant de ses subsides - le récit du génocide des Tutsi serait aujourd'hui totalement différent. Peut-être même que l'on n'en parlerait plus du tout. La forte personnalité de Paul Kagame - à qui on ne peut en outre dénier un formidable sens de I'histoire - lui a rendu la tâche encore plus difficile.

Elle l'était d'autant plus que, dans la société française elle-même, des lanceurs d'alerte avant la lettre avaient très tôt fait du génocide contre les Tutsi au Rwanda le combat de leur vie. Même si la présence la plus mémorable restera à jamais celle, tutélaire, de FrançoisXavier Verschave, fondateur de Survie, l'image emblématique de cet engagement citoyen pourrait bien être celle de Jean Carbonare face au présentateur du 20 heures de France 2, Bruno Masure, le 24 janvier 1993 : « Ce qui nous a frappé au Rwanda, lui explique-t-il, c'est à la fois l'ampleur, la systématisation, l'organisation même de ces massacres... II y a un mécanisme qui se met en route. On a parlé de purification ethnique, de génocide, de crimes contre I'humanité ». Et se faisant plus précis: "Notre pays, qui soutient militairement et financièrement ce système, a une responsabilité. Notre pays peut, s'il le veut, peser sur cette situation ». Et presque en larmes, la voix brisée : "Vous aussi monsieur Masure, vous pouvez faire quelque chose! Vous devez faire quelque chose... pour que cette situation change, parce qu'on peut la changer si on veut. »

Ce moment de télévision est entré dans la légende mais aucun décideur n'a voulu tirer les conséquences de l'exceptionnel cri de détresse de Jean Carbonare, à l'époque président de Survie.

Parmi les voix qui se sont élevées après le génocide au nom d'une conception moins patriotarde de l'honneur de la France, celle de Jacques Julliard - pourtant peu connu pour

\footnotetext{
${ }^{5}$ Chomsky Noam ([2005] 2006), La Doctrine des bonnes intentions. Entretiens avec David Barsamian, Paris, Fayard.
} 
son intérêt pour le Rwanda - a aujourd'hui une résonance toute particulière. Dès avril 1998, il écrit dans sa chronique du Nouvel Observateur: "Se posera un jour, n'en doutons pas, la question de la responsabilité de la France, François Mitterrand étant président de la République, dans le génocide des Tutsis du Rwanda en 1994. La France n'a pas commis le crime, mais elle a armé les bras de futurs tueurs qui ne cachaient pas leurs intentions ${ }^{6}$. »

Dès 1997, paraît le livre de Mehdi Bâ, Rwanda, un génocide français ${ }^{7}$, au titre tout à fait explicite; avec son ouvrage très fouillé et au ton rageur La France au coeur du génocide des Tuts $i^{8}$, Jacques Morel est encore moins ambigu; et s'il faut évoquer Jean-Paul Gouteux, auteur de La Nuit rwandaise, ou des films tels que Tuez-les tous ! ${ }^{10}$ et Un cri d'un silence inoui ${ }^{11}$, mention spéciale doit être faite de ce que l'on a fini par appeler avec Michel Sitbon et Mehdi Bâ « la révolution saint-exupérienne ${ }^{12}$ ».

Patrick de Saint-Exupéry ne s'attendait certes pas, en se rendant au Rwanda pour Le Figaro, un journal peu enclin aux positions spectaculaires, à ce que l'expérience change du tout au tout le cours de son existence. Les articles qu'il a envoyés en son temps ne pouvant suffire à dire le choc émotionnel et intellectuel éprouvé, il ne cesse de revenir sur le sujet, de $L^{\prime \prime I n a v o u a b l e . ~ L a ~ F r a n c e ~ a u ~ R w a n d a ', ~ a ̀ ~ C o m p l i c e s ~ d e ~ l ' i n a v o u a b l e ~}{ }^{14}$ en passant par la revue $X X I$ jusqu'à tout récemment $L a$ Traversée, journal de voyage à la fois grave et enjoué où il entreprend de démolir méthodiquement la thèse du deuxième génocide, supposé avoir été commis dans l'Est du Congo par un FPR revanchard et ivre de sang ${ }^{15}$.

Au final, sans l'opiniâtreté de groupes tels que Survie et le Collectif des Parties civiles pour le Rwanda, ou de fortes individualités, il serait aujourd'hui encore presqu'impossible de distinguer les victimes rwandaises de leurs bourreaux et donc d'écrire la véritable histoire du génocide des Tutsi. II n'y aurait pas eu, vingt-sept ans après, de rapport Duclert.

On découvre à la lecture de celui-ci que des fonctionnaires français travaillant sur le dossier ont, entre octobre 1990 et juillet 1994 et même un peu avant dans le cas du colonel René Galinié, fait de leur mieux pour éviter au peuple rwandais un bain de sang et à leur pays le déshonneur d'y avoir été pour quelque chose.

C'est le cas du ministre Pierre Joxe peu impressionné par les postures monarchiques, finalement assez dérisoires, de Mitterrand; Yannick Gérard, ambassadeur de France en Ouganda, le colonel Patrice Sartre et Antoine Anfré, "rédacteur Rwanda » au Quai d'Orsay, sont aussi de ceux-là. Le dernier nommé, Anfré, débarqué à l'époque en raison de ses vues

\footnotetext{
6 Julliard Jacques, chronique hebdomadaire, Nouvel Observateur, avril 1998.

7 Bâ Mehdi (1997), Rwanda, un génocide français, Paris, L'Esprit frappeur.

8 Morel Jacques (2010), La France au cour du génocide des Tutsi, Paris, Izuba/L'Esprit frappeur.

9 Gouteux Jean-Paul (2002), La Nuit rwandaise. L'implication française dans le dernier génocide du siècle, Paris, Izuba/L'Esprit frappeur. Pour lui rendre hommage une revue sur le génocide des Tutsi au Rwanda porte ce titre.

10 Glucksmann Raphaël, Hazan Pierre et Mezerette Pierre (2004), Tuez-les tous! (Rwanda: Histoire d'un génocide " sans importance »), Dum Dum Films, La Classe américaine.

11 Lainé Anne (2003), Rwanda, un cri d'un silence inouï, France 5, Little Bear Productions, Palindromes.

12 Michel Sitbon, cité par Stephen Smith dans "L'infamante accusation de "complicité" de la France est portée sans preuve », Le Monde, 17 avril 2004, en ligne. URL : https://www.lemonde.fr/archives/article/2004/04/17//-infamante-accusation-de-complicite-dela-france-est-portee-sans-preuves_361502_1819218.html (consulté le 11 octobre 2021).

13 Saint-Exupéry Patrick (de) (2004), L'Inavouable. La France au Rwanda, Paris, Les Arènes.

14 Saint-Exupéry Patrick (de) (2009), Complices de l'inavouable. La France au Rwanda, Paris, Les Arènes.

15 Saint-Exupéry Patrick (de) (2021), La Traversée. Une odyssée au ccur de l'Afrique, Paris, Les Arènes.
} 
dissidentes, vient d'être nommé ambassadeur à Kigali. À l'heure de l'apaisement entre les deux pays, c'est tout un symbole.

Il est de même souligné à plusieurs reprises que la Direction générale de la Sécurité extérieure (DGSE) n'a cessé, au cours de ces années, de donner aux politiques des informations en totale contradiction avec la doxa mitterrandienne. II est ainsi écrit dans le rapport Duclert qu'au début de Turquoise, la DGSE avait averti dans une note: "Le danger est grand pour la France [...] de passer pour complice de l'actuel gouvernement rwandais ${ }^{16}$. » Et, citant plus loin un autre bulletin de renseignements : "La DGSE affirme dès le 2 mai que le FPR est "très certainement étranger à l'attentat qui a coûté la vie au président Habyarimana", attentat qu'elle attribuera aux extrémistes hutu ${ }^{17}$. "Parmi les militaires, le colonel Patrice Sartre n'hésite pas à donner en annexe de son rapport de fin de mission une liste de noms de génocidaires présumés, qui pourrait, selon lui :

faire l'objet d'une enquête interne française, afin de déterminer le plus clairement possible quels responsables français, ayant été en contact régulier avec les personnes mises en cause, peuvent faire l'objet d'une critique, au moins médiatique, pour complicité avec la planification du génocide. $^{18}$

Attaché de défense à Kigali entre juin 1988 et juillet 1991, le colonel René Galinié est crédité dans le rapport d'une exceptionnelle clairvoyance. Voici le résumé qui y est fait de "l'évolution de son regard » à partir de la première offensive du FPR en octobre 1990. Le 8 octobre, il signale une répression organisée à Kigali, l'arrestation de suspects "parfois fusillés ". II note surtout que "cette chasse pourrait, en cas d'aggravation, dégénérer en tueries ». Le 10 octobre, son message du jour mentionne sa crainte " que ce conflit finisse par dégénérer en guerre ethnique ». II rapporte d'autre part que le MRND (Mouvement révolutionnaire national pour le développement), "parti unique », "semble reprendre en main le pays hors de la zone des combats". Le 13 octobre, le même témoin constate que "les paysans hutus organisés par le MRND ont intensifié la recherche des Tutsis suspects dans les collines; des massacres sont signalés dans la région de Kibilira, à 20 kilomètres nord-ouest de Gitarama ${ }^{19}$ ».

Un tel sens de l'anticipation coûte à Galinié son poste ainsi qu'au général Varret qui, lui, " s'opposait à l'intervention directe de l'Élysée dans la coopération et luttait pour conserver la maîtrise de la coopération militaire, comme les institutions lui en donnaient la mission ${ }^{20} »$.

L'attitude raisonnable de ces personnalités civiles et militaires renforce, a contrario, l'opinion selon laquelle, faute de mettre en accusation l'État français, on devrait au moins juger François Mitterrand et tous ceux qui, à partir de l'Élysée ou de certaines officines ministérielles, ont une responsabilité directe dans le massacre de plus d'un million de civils innocents. Georges Martres et Jean-Michel Marlaud, qui se sont succédés comme ambassadeurs à Kigali savaient très bien ce qu'ils faisaient : il leur est arrivé de dissimuler ou

\footnotetext{
16 Commission de recherche sur les archives françaises relatives au Rwanda et au génocide des Tutsi (2021), La France, le Rwanda et le génocide des Tutsi (1990-1994), Paris, Armand Colin, p. 702. Ci-après Rapport Duclert.

17 Ibid.

18 Rapport Duclert, p. 890.

19 Rapport Duclert, p. 774.

20 Rapport Duclert, p. 754.
} 
de déformer des faits pour que le soutien de la France à Habyarimana ne soit jamais remis en cause. Ils étaient pourtant bien au courant des massacres du Bugesera ou de celui des Bagogwe - perpétrés par leur protégé au moment même où ils portaient son régime à bout de bras. Le général Quesnot, chef d'état-major particulier du président Mitterrand, Bruno Delaye, conseiller en charge des questions africaines et le secrétaire général de l'Élysée, Hubert Védrine, très souvent cités dans le rapport Duclert, ont pesé encore plus lourd dans la prise des décisions ayant favorisé le crime et protégé les criminels. Quant au ministre Juppé, il est souvent rappelé qu'il est le premier à avoir utilisé le mot "génocide ». Cela est vrai mais c'était le 16 mai, cinq semaines et au moins un demi-million de morts plus tard. Il est du reste à noter que seulement quatre jours plus tôt, le même ministre français des Affaires étrangères déclarait à Washington: "Nous n'avons pas laissé le Rwanda à l'abandon pendant toutes ces années, nous avons essayé de tout faire pour réconcilier les tribus, puisqu'il s'agit en fait d'un combat tribal ${ }^{21}$. „ Si on en juge par les actes qu'il a posés au nom de son pays, ce point de vue nourri de stéréotypes racistes est resté jusqu'au bout son intime conviction. Et si son mea culpa d'avril dernier a suscité un certain émoi, c'est surtout parce que Juppé a toujours si fermement nié toute implication de la France dans le génocide de 1994. Mais sa contrition est assez cyniquement simulée. Il écrit par exemple : « Nous n'avons pas imaginé que nos forces déployées pour assurer la protection de nos ressortissants, auraient pu, à condition d'avoir le soutien des parachutistes belges, des commandos italiens, des marines américains présents au Burundi, tous associés aux casques bleus, s'opposer aux tueurs, protéger les victimes ${ }^{22}$.» II est étrange qu'un homme politique réputé particulièrement intelligent s'imagine pouvoir nous faire croire aujourd'hui que Paris aurait eu besoin des Belges, des Italiens, des Américains et des casques bleus pour mettre fin aux massacres. Rien n'est plus faux. Le régime génocidaire de Kigali, tenu à bout de bras par le gouvernement français, lui obéissait au doigt et à l'œil. L'ancien ministre des Affaires étrangères, qui a d'ailleurs reçu au Quai d'Orsay son homologue Jérôme Bicamumpaka au plus fort des tueries, le sait mieux que quiconque. Cela rend la faute morale plus lourde et presque plus incompréhensible.

De toute façon, Mitterrand n'avait besoin de l'avis de personne pour agir comme il l'a fait. II ne donne à aucun moment l'impression d'être effleuré par le moindre doute. II n'y a pas que de la vanité dans la prétention de certains politiques à mieux connaître que quiconque l'histoire de l'humanité : ils veulent aussi nous persuader qu'à l'instant même où ils scrutent le passé, ils ont le regard tourné vers l'avenir. Mitterrand a toujours eu cette posture et on est d'autant plus choqué de constater que sur le Rwanda, sa ligne de pensée est très exactement celle des artisans de la «révolution sociale hutu » de la fin des années 1950. Pour Mitterrand aussi il y a une sorte d'équivalence absolue - du moins « dans ces pays-là » - entre démographie et démocratie. La principale vertu des Hutu c'est, à ses yeux, d'être "le peuple majoritaire» et, pas plus que les membres de son premier cercle, le président français ne peut imaginer un Rwanda « dirigé par les Tutsi » sans qu'y survienne un sanglant chaos. Le Rwanda d'aujourd'hui fournit la preuve qu'il n'est pas bien difficile, même

\footnotetext{
21 Rapport Duclert, p. 941.

22 Alain Juppé, « Nous n'avons pas compris qu'un génocide ne pouvait supporter des demi-mesures », Le Monde, 7 avril 2021 , en ligne. URL : https://www.lemonde.fr/idees/article/2021/04/07/alain-juppe-sur-le-rwanda-nous-n-avons-pas-compris-qu-un-genocidene-pouvait-supporter-des-demi-mesures_6075875_3232.html (consulté le 11 octobre 2021).
} 
après un génocide, de bâtir un État non-fondé sur l'ethnisme dans lequel l'avait piégé l'occupant belge pour mieux asseoir sa propre domination.

Tout cela étant dit, pour un Sénégalais, citoyen d'un "pays du champ », le rapport Duclert est l'occasion d'une fructueuse chasse au trésor sur le fonctionnement de la Françafrique. Par exemple, la forte relation personnelle entre le locataire de l'Élysée et chacun de ses laquais africains est une donnée constante. Bokassa appelait Giscard « mon parent » et de Gaulle «mon papa » et tout en pillant de concert l'Afrique, Chirac, Sassou-Nguesso et Bongo-père - ou d'autres - se comportaient comme une bande de joyeux drilles, assez virils et machos, mais incapables de faire du mal à une mouche. De même, sans être aussi exubérante, "l'amitié » ostentatoire entre Habyarimana et Mitterrand est déterminante dans la lecture que ce dernier fait de la situation au Rwanda. Son homologue en profite pour contourner habilement les circuits de décision classiques et obtenir presque tout ce qu'il veut de la France.

Ce n'est pas tout, bien évidemment.

On voit se dessiner au fil des pages du rapport Duclert la vision globalisante des stratèges de la Françafrique. Comme tous les États-clients de la Françafrique le Rwanda n'a, vu de Paris, aucune existence singulière, ses réalités sont perçues en fonction d'un vaste projet de domination à long terme - pour ne pas dire éternelle. C'est ce qui amène le général Quesnot à écrire que laisser tomber Habyarimana reviendrait à envoyer « un signal très négatif à nos alliés africains ${ }^{23}$ », avant de se montrer encore plus précis dans une note à Roland Dumas :

Les inconvénients sont clairs: notre départ serait perçu comme un abandon de nos amis, comme un échec de la France (nous avons dit: nous ne laisserons pas prendre Kigali). La crédibilité de notre politique africaine serait dans une large mesure atteinte. Enfin, s'il y a des massacres et cela est probable, nous risquerions d'en être tenus pour responsables. ${ }^{24}$

Il attire aussi I'attention de Mitterrand sur les enjeux linguistiques de la question en ces termes :

Si nous ne trouvons pas le moyen de pression suffisant pour arrêter Museveni, qui bénéficie du soutien britannique implicite, le front de la francophonie sera durablement mis à mal et compromis dans cette région. Contrairement à l'évolution historique actuelle, une ethnie tutsie minoritaire s'assurera du pouvoir par la force sur un ensemble régional Ouganda-RwandaBurundi. ${ }^{25}$

Hubert Védrine a résumé tout cela en une formule apparemment anodine («l'engagement global de sécurité de la France ») mais qui est en réalité une justification $a$ priori des plus sanglantes équipées néocoloniales françaises. Appliquée au cas particulier du Rwanda, elle signifiait que tout devait être fait pour stopper les ennemis armés d'un régime sous protection française, des gens d'ailleurs bizarres, parlant anglais et venus d'un pays anglophone, l'Ouganda.

\footnotetext{
23 Rapport Duclert, p. 232.

24 Rapport Duclert, pp. 234-235.

25 Rapport Duclert, p. 242.
} 
C'est cela qui explique l'imposante taille des archives françaises sur le Rwanda ainsi que le rythme infernal des « conseils restreints » et réunions ordinaires qui lui sont consacrés.

À l'ère des réseaux sociaux, le rapport Duclert est beaucoup plus médiatisé que les précédents. Il faut dire aussi qu'il a été commandité à des universitaires par un président qui n'avait que 16 ans pendant le génocide. Sa conclusion a été répétée partout et beaucoup n'en retiendront rien d'autre: La France n'est pas complice de ce génocide même si elle s'est "longuement investie au côté d'un régime qui encourageait des massacres racistes » puis " est demeurée aveugle face à la préparation d'un génocide par les éléments les plus radicaux de ce régime ${ }^{26}$ ». II y a donc lieu de relever des "responsabilités, lourdes et accablantes $^{27}$ » de la France imputable en particulier à François Mitterrand. Décider du rôle d'un État dans un génocide ne présentant aucune ambiguïté ne pouvait être chose aisée. II est logique de supposer que chaque mot de la conclusion du rapport a été négocié, sans doute parfois pied à pied, avec l'autorité politique. Dans le monde tel qu'il va, les membres de la commission de recherche ne pouvaient raisonner en dehors du réel. II est toutefois moins compréhensible qu'après avoir mis en évidence, parfois avec une pointe de mépris, l'action néfaste du président français et de certains de ses collaborateurs, la commission n'ait pas conclu à leur complicité avec des hommes comme le colonel Théoneste Bagosora et I'historien Ferdinand Nahimana considérés comme deux des principaux architectes de l'apocalypse.

Mais la manifestation de toute la vérité sur un génocide est toujours une affaire de longue haleine. Elle ne peut surgir que lentement, dans la douleur et dans la honte. Qui aurait pu imaginer il y a seulement dix ans que deux présidents français se rendraient un jour au mémorial de Gisozi, I'un pour admettre, même du bout des lèvres, des " erreurs ", l'autre pour passer aux aveux - enfin presque... - dans un discours style Malraux ? "Le génocide franco-africain. Faut-il juger les Mitterrand? "C'était, dès 1994, le titre d'un ouvrage de Pascal Krop ${ }^{28}$. La question a sans doute paru farfelue à beaucoup à l'époque. Elle ne l'est plus et en soi cela est un grand pas en avant. Le travail d'introspection sur le Rwanda va sans nul doute se poursuivre en France, ce qui signifie que ce troisième rapport sur le génocide des Tutsi au Rwanda ne sera pas le dernier. On peut dire en termes familiers qu'à l'échelle de I'histoire, Mitterrand père et fils, Quesnot, Martres, Védrine et quelques autres ne perdent rien pour attendre.

Boubacar Boris Diop écrivain (Sénégal)

\section{Bibliographie}

BA Mehdi (1997), Rwanda, un génocide français, Paris, L'Esprit frappeur.

\footnotetext{
${ }^{26}$ Rapport Duclert, p. 972.

27 Rapport Duclert, p. 973.

28 Krop Pascal (1994), Le Génocide franco-africain. Faut-il juger les Mitterrand? Paris, Jean-Claude Lattès.
} 
BANCEL Nicolas (2002), « Les médias français face au Rwanda. De l'intervention française de 1990 au génocide », Africultures, 30, en ligne. URL: http://africultures.com/les-medias-francais-face-aurwanda-1471/ (consulté le 11 octobre 2021).

ChOMSky Noam ([2005] 2006), La Doctrine des bonnes intentions. Entretiens avec David Barsamian, Paris, Fayard.

COMMISSION DE RECHERCHE SUR LES ARCHIVES FRANÇAISES RELATIVES AU RWANDA ET AU GENOCIDE DES TUTSI (2021), La France, le Rwanda et le génocide des Tutsi (1990-1994), Paris, Armand Colin.

Glucksmann Raphaël, Hazan Pierre et Mezerette Pierre (2004), Tuez-les tous ! (Rwanda : Histoire d'un génocide "sans importance »), Dum Dum Films, La Classe américaine.

Gouteux Jean-Paul (2002), La Nuit rwandaise. L'implication française dans le dernier génocide du siècle, Paris, Izuba/L'Esprit frappeur.

KROP Pascal (1994), Le Génocide franco-africain. Faut-il juger les Mitterrand?, Paris, Jean-Claude Lattès.

LaINe Anne (2003), Rwanda, un cri d'un silence inouï, France 5, Little Bear Productions, Palindromes.

MOREL Jacques (2010), La France au coeur du génocide des Tutsi, Paris, Izuba/L'Esprit frappeur.

SAINT-EXUPeRY Patrick (de) (2004), L'Inavouable. La France au Rwanda, Paris, Les Arènes. (2009), Complices de l'inavouable. La France au Rwanda, Paris, Les Arènes. (2021), La Traversée. Une odyssée au cour de l'Afrique, Paris, Les Arènes. 